GA-A24340

\title{
ELECTRON CYCLOTRON CURRENT DRIVE IN DIII-D: EXPERIMENT AND THEORY
}

\author{
by \\ R. PRATER, C.C. PETTY, T.C. LUCE, R.W. HARVEY, M. CHOI, \\ R.J. LA HAYE, Y.-R. LIN-LIU, J. LOHR, M. MURAKAMI, \\ M.R. WADE, and K.-L. WONG
}




\section{DISCLAIMER}

This report was prepared as an account of work sponsored by an agency of the United States Government. Neither the United States Government nor any agency thereof, nor any of their employees, makes any warranty, express or implied, or assumes any legal liability or responsibility for the accuracy, completeness, or usefulness of any information, apparatus, product, or process disclosed, or represents that its use would not infringe privately owned rights. Reference herein to any specific commercial product, process, or service by trade name, trademark, manufacturer, or otherwise, does not necessarily constitute or imply its endorsement, recommendation, or favoring by the United States Government or any agency thereof. The views and opinions of authors expressed herein do not necessarily state or reflect those of the United States Government or any agency thereof. 


\title{
ELECTRON CYCLOTRON CURRENT DRIVE IN DIII-D: EXPERIMENT AND THEORY
}

\author{
by \\ R. PRATER, C.C. PETTY, T.C. LUCE, R.W. HARVEY, ${ }^{\star}$ M. CHOI, \\ R.J. LA HAYE, Y.-R. LIN-LIU, J. LOHR, M. MURAKAMI, ${ }^{\dagger}$ \\ M.R. WADE, ${ }^{\dagger}$ and K.-L. WONG ${ }^{\ddagger}$
}

This is a preprint of a paper to be presented at the 15th

Topical Conference on Radio Frequency Power in Plasmas, Moran,Wyoming, May 19-21, 2003 and to be published in the Proceedings.

*CompX, Del Mar, California.

tOak Ridge National Laboratory, Oak Ridge, Tennessee.

‡Princeton Plasma Physics Laboratory, Princeton, New Jersey.

\author{
Work supported by \\ the U.S. Department of Energy \\ under Contract Nos. DE-AC03-99ER54463, DE-AC05-000R22725, \\ DE-AC02-76CH03073, and DE-FG03-99ER54541
}

GENERAL ATOMICS PROJECT 30033

JULY 2003 


\title{
Electron Cyclotron Current Drive in DIII-D: Experiment and Theory
}

\author{
R. Prater, C.C. Petty, T.C. Luce, R.W. Harvey, ${ }^{\mathrm{a}}$ M. Choi, R.J. La Haye, \\ Y.-R. Lin-Liu, J. Lohr, M. Murakami, ${ }^{b}$ M.R. Wade, ${ }^{b}$ K.-L. Wong ${ }^{c}$ \\ General Atomics, P.O. Box 85608, San Diego, California 92186-5608 \\ ${ }^{a}$ CompX, Del Mar, California. \\ "Oak Ridge National Laboratory, Oak Ridge, Tennessee. \\ 'Princeton Plasma Physics Laboratory, Princeton, New Jersey.
}

\begin{abstract}
Experiments on the DIII-D tokamak in which the measured off-axis electron cyclotron current drive has been compared systematically to theory over a broad range of parameters have shown that the Fokker-Planck code CQL3D provides an excellent model of the relevant current drive physics. This physics understanding has been critical in optimizing the application of ECCD to high performance discharges, supporting such applications as suppression of neoclassical tearing modes and control and sustainment of the current profile.
\end{abstract}

\section{INTRODUCTION}

Electron cyclotron heating $(\mathrm{ECH})$ and current drive (ECCD) have been intensively studied in experiments on the DIII-D tokamak [1-3]. The key objectives are to develop and validate a predictive computational model which will be applicable to next-step devices as well as present-day experiments and to use that model to optimize the many applications of EC power in DIII-D as part of the Advanced Tokamak program. Full validation of the model requires that the relevant physics parameters be recognized and varied in a systematic way in experiments, and that the measurements of the magnitude and profile of driven current be determined and compared with the predictions of the model. The physics model is implemented in the ray tracing code TORAY-GA [4,5] and in the quasilinear Fokker-Planck code CQL3D [6]. This model has been used successfully to obtain the best possible performance of the ECH system on DIII-D in experiments on stabilizing $m=3 / n=2[7-9]$ and $m=2 / n=1$ [10] neoclassical tearing modes and to improve and sustain the current profile which has led to improved tokamak performance [11].

\section{EXPERIMENTAL APPROACH}

Detailed current drive experiments were carried out on the DIII-D tokamak [12]. For these experiments plasmas have major radius $1.7 \mathrm{~m}$, minor radius $0.6 \mathrm{~m}$, elongation typically 1.8 , and toroidal field up to $2.1 \mathrm{~T}$. The EC system uses up to five 
gyrotrons in these experiments to generate up to $2.5 \mathrm{MW}$ incident on the plasma for pulses up to $2 \mathrm{~s}$ [13]. The $110 \mathrm{GHz}$ frequency of the gyrotrons is resonant with the second harmonic EC frequency at a field of $1.96 \mathrm{~T}$. The EC launcher is placed at the outboard side about $0.7 \mathrm{~m}$ above the midplane, and most of the launchers can steer the beam independently in both the vertical (poloidal) and horizontal (toroidal) directions [14]. This launching system provides great experimental freedom to control the location and width of the profile of driven current, as both the radial location and the $\mathrm{n}_{\|}$can be determined by the launch angles for a given equilibrium and kinetic profiles. The physics of ECCD is studied in these experiments through orthogonal scans, such as of minor radius with $n_{\|}$fixed or of $n_{\|}$with the minor radius fixed.

The critical diagnostic for measurement of the magnitude and profile of ECCD is the motional Stark effect system [15], which measures the pitch angle of the magnetic field at $\approx 5 \mathrm{~cm}$ intervals across the plasma outer midplane. Time-dependent plasma simulations are used for a range of radial locations, magnitudes, and profile widths to determine the profile of driven current which best matches the actual measurements [2]. Thus, the effects of the back emf generated by the localized ECCD can be taken into account, since in many cases the toroidal electric field is not fully equilibrated.

The dimensionless current drive efficiency $\zeta=\left(\mathrm{e}^{3} / \varepsilon_{0}^{2}\right) \mathrm{n}_{\mathrm{e}} \mathrm{I}_{\mathrm{EC}} \mathrm{R} / \mathrm{T}_{\mathrm{e}} \mathrm{P}_{\mathrm{EC}}$ is used as a figure-of-merit to characterize the effectiveness of the EC power in driving current [3]. This efficiency follows from a natural normalization of the current and power density, and it includes the theoretical dependencies on plasma density and temperature [aside from the slowly-varying $\ln (\Lambda)$ factor] which would be expected of any current drive scheme which acts on the electron distribution near the thermal velocity. Hence, deviations of $\zeta$ from a constant are due to interaction with a different part of velocity space or to an effect like trapping of electrons in the magnetic well. Plotting $\zeta$ in a radial scan illustrates the dependence of the physics of off-axis ECCD without the expected but uninteresting fall-off in current due to decreasing $\mathrm{T}_{\mathrm{e}}$.

The driven currents can be compared with the currents theoretically expected. The relevant theory has been encapsulated in the ray tracing code TORAY-GA and in the quasilinear Fokker-Planck code CQL3D. TORAY-GA uses the cold plasma dispersion relation to determine the ray trajectories and relativistic models for the wave polarization and absorption. It also uses the Cohen Green's function model [5] for calculating the driven current. The ray trajectories and polarizations from TORAY-GA can be input to CQL3D to calculate currents including quasilinear effects due to higher power densities and the effect of $\mathrm{E}_{\mid}$on the distribution function. CQL3D uses a collision operator which preserves momentum in electron-electron collisions, unlike that in TORAY-GA. In practice, all of these effects can be important.

\section{COMPARISON OF EXPERIMENT AND THEORY}

Excellent agreement of the measured current with the calculated current is found over the DIII-D database of about 80 discharges [1]. This agreement is illustrated in Fig. 1, which shows the measured current vs the current calculated by the CQL3D code, including the effects of $E_{\|}$. The dashed line in Fig. 1 represents perfect agreement, and the error bars of most data points intersect that line. Note that the data base includes many cases with negative driven current which are also in good agree- 
ment with theory. In those cases the quasilinear effects on the resistivity given finite positive $\mathrm{E}_{\|}$increase the calculated ECCD rather than decrease it, but still the agreement with theory is excellent.

The data points illustrated in Fig. 1 represent a range of temperatures, densities, minor radii $\rho, n_{\|}$, and $\theta_{p}$, where the $\theta_{p}$ is the angular variable along a flux surface going from 0 at the outboard midplane to $180 \mathrm{deg}$ at the inboard midplane, and all quantities are measured at the location of the wave-particle interaction. Subsets of these data can then be replotted as single parameter scans, where all parameters but the independent parameter are held fixed. In Fig. 2 the dimensionless current drive efficiency is plotted against $\mathrm{n}_{\|}$for two such scans. In these scans, the density and temperature dependences are categorized jointly as $\beta_{\mathrm{e}}$, which is a good characterization of the behavior even though the physics of the dependences on $\mathrm{n}_{\mathrm{e}}$ and $\mathrm{T}_{\mathrm{e}}$ is slightly different. In Fig. 2 the two curves are for low $\beta_{\mathrm{e}} \mathrm{L}$-mode discharges, the principle difference being that the upper curve is for an interaction near the inboard midplane where the magnetic well depth is minimum, while the lower curve is for an interaction near the top of the flux surface where the well depth is moderate, both cases being for $\rho=0.3$. Comparing these cases, it can be seen that the efficiency saturates for sufficiently large $n_{\|}$, but that the saturation level is larger for the case with smaller well depth (i.e., $\theta_{p}=175 \mathrm{deg}$ ). The agreement between the data points and the CQL3D calculations, including $\mathrm{E}_{\|}$ effects, shows that the model has very little systematic variance from the measurements for this cut through the data.

The data in Fig. 2 are consistent with the physically intuitive idea that trapping of the current-carrying electrons reduces the efficiency of ECCD. Figure 3 shows another case illustrating this behavior. The unfilled points in Fig. 3 show that the efficiency at low fixed $\beta_{\mathrm{e}}$, fixed $\theta_{\mathrm{p}}$ near $90 \mathrm{deg}$, and fixed $n_{\|}$drops as the normalized minor radius increases and the well depth increases. However, the filled points for the same conditions but higher $\beta_{\mathrm{e}}$ show that the decrease with radius is much smaller,

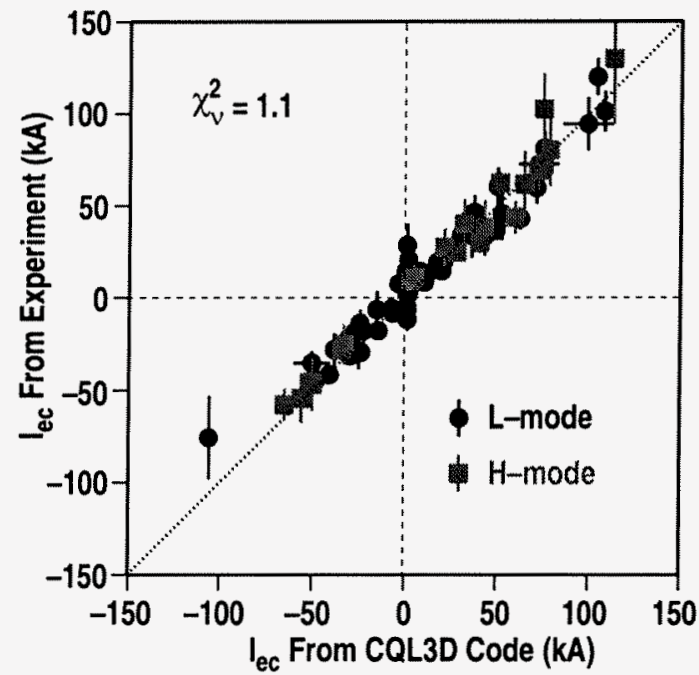

FIGURE 1. The measured ECCD current for the entire database of discharges in DIII-D versus the current calculated by the CQL3D Fokker-Planck code including the effect of the parallel electric field. The circular data points are for L-mode and the squares are for $\mathrm{H}$-mode discharges.

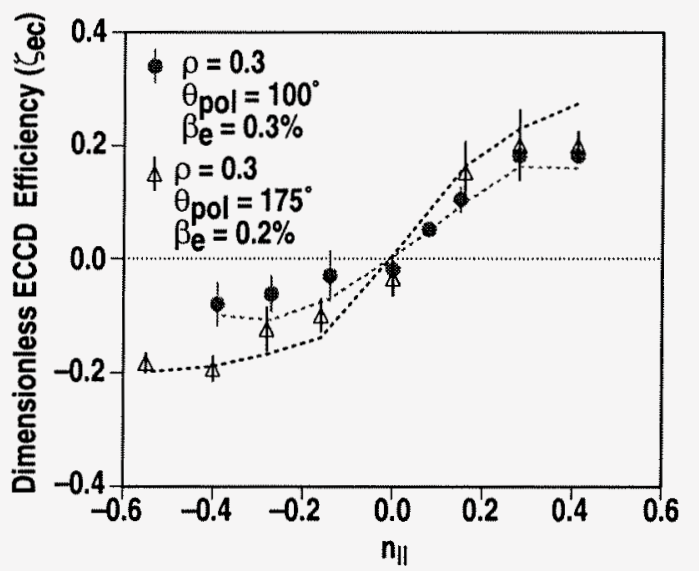

FIGURE 2. Dimensionless current drive efficiency versus parallel index of refraction for discharges with ECCD at $\rho=0.3$, low $\beta_{\mathrm{e}}(<0.3 \%)$, and $\theta_{\mathrm{pol}}=175 \mathrm{deg}$ (triangles) and $\theta_{\mathrm{pol}}=100 \mathrm{deg}$ (circles). The dashed lines represent the values from calculations using CQL3D. 
and at $\rho=0.4$ the dimensionless efficiency for the higher $\beta_{\mathrm{e}}$ case is double that of the lower $\beta_{\mathrm{e}}$ case.

The behavior of the efficiency with $\beta_{\mathrm{e}}$ must be understood in the context of the competition between the Fisch-Boozer current (FB) and the Ohkawa current (OK), the two elements comprising the generation of ECCD [14]. The FB current arises when electrons with one sign of $\mathrm{v} \|$ are accelerated by the EC waves to higher energy. At the higher energy, the electrons pitch-angle scatter more slowly than the electrons at the lower energy. In the limit of a steady-state process, this leaves an excess of electrons with that sign of $\mathrm{v} \|$. The OK current arises when electrons with one sign of $\mathrm{v}_{\|}$are accelerated in $\mathrm{v}_{\perp}$ into the trapped region of velocity space. These electrons in the trapped region symmetrize rapidly, leaving behind a deficit at the sign of $\mathrm{v} \|$. So the toroidal direction of the two current components is opposite, and the net current depends on the details of the balance. For this reason, no simple scaling is possible for estimating the decrement of the net current drive efficiency of off-axis ECCD.

The TORAY-GA code calculates this net current drive. For the relevant geometry of Fig. 4(a), the data along the central ray are plotted in Fig. 4(b). This figure shows the normalized power deposited in the plasma per unit ray length $(1 / \mathrm{P}) \mathrm{dP} / \mathrm{ds}$ and the quantity $\left(\omega / 2 \Omega_{\mathrm{e}}\right)-1$, where $\omega$ is the applied frequency and $\Omega_{\mathrm{e}}$ is the local electron cyclotron frequency. Also shown is the calculated driven toroidal current per unit ray length, $\mathrm{dI} / \mathrm{ds}$. The case shown in Fig. 4 is for low $\beta_{\mathrm{e}}$, and hence relatively weak damping, as Fig. 4(b) shows that the ray crosses the cold resonance where $\left(\omega / 2 \Omega_{e}\right)-1=0$ with significant power still remaining.

The behavior of $\mathrm{dI} / \mathrm{ds}$ in Fig. 4(b) is easily understood from the discussion of FB and $\mathrm{OK}$ current drive discussed above. As the ray propagates, it enters the region close enough to the cold resonance that power is absorbed but still with $\left(\omega / 2 \Omega_{\mathrm{e}}\right)-1>0$. To satisfy the relativistic cyclotron resonance for the applied $\omega$ and wavenumber $k \|$,

$$
\frac{v_{\perp}^{2}}{c^{2}}=1-\frac{v_{\|}^{2}}{c^{2}}-\left(\frac{\omega}{2 \Omega_{e}}\right)^{2}\left[1-2 n_{\|}\left(\frac{v_{\|}}{c}\right)+n_{\|}^{2}\left(\frac{v_{\|}^{2}}{c^{2}}\right)\right],
$$

the $\mathrm{v} \|$ must be large relative to the thermal velocity. This resonance in velocity space corresponding to this location in physical space is shown in Fig. 4(c) for the central ray of the ray bundle. The wave-particle interaction takes place along this resonance curve, which does not closely approach the T-P boundary. Because of this relatively weak interaction with the trapped region of velocity space, the FB current is strongly dominant and $\mathrm{dI} / \mathrm{ds}$ is large and positive. As the wave approaches closer to the resonance, 
(a)

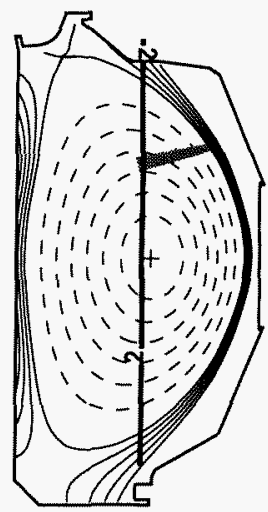

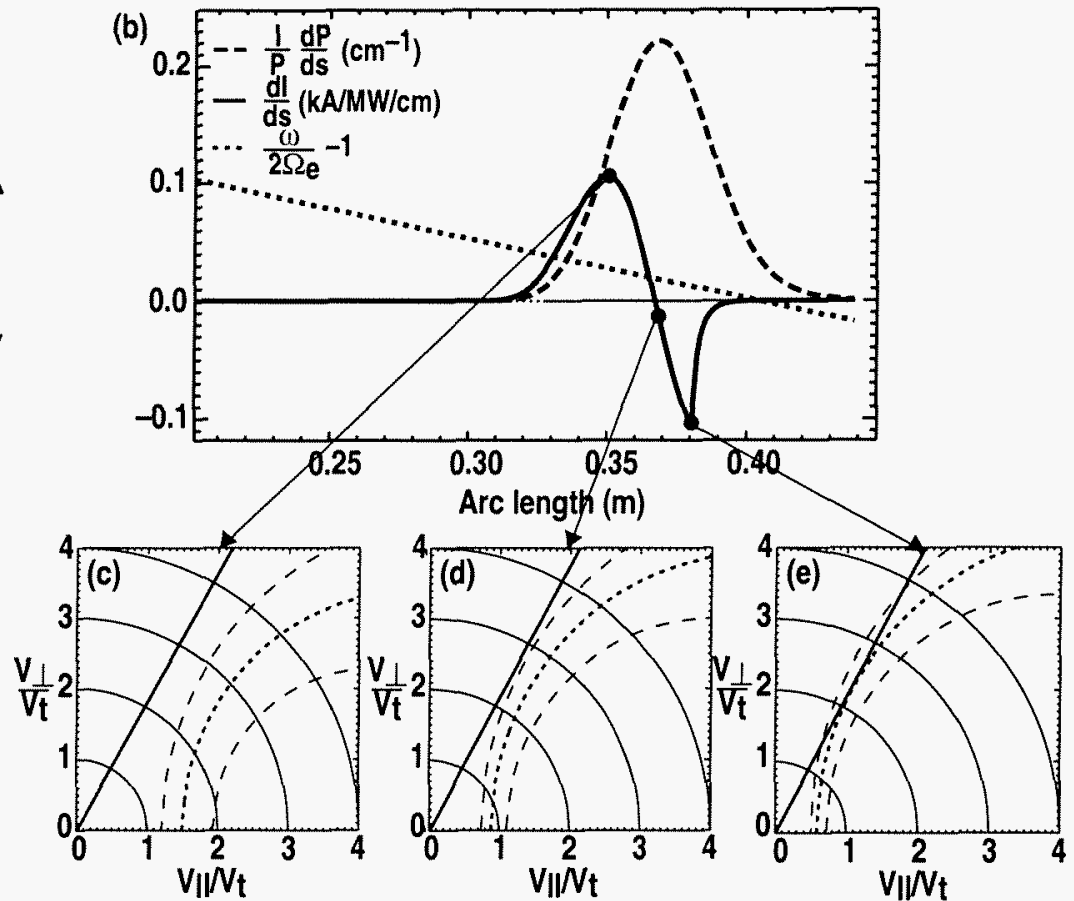

FIGURE 4. (a) Geometric arrangement of rays; (b) $(1 / \mathrm{P})(\mathrm{dP} / \mathrm{ds}), \mathrm{d} / \mathrm{ds}$, and $\omega / 2 \Omega_{\mathrm{e}}-1$ as a function of ray are length for the central ray of the bundle shown in (a) as calculated by the TORAY-GA code; (c-e) resonance curve (dotted) and trapped-passing boundary (solid curve) in normalized velocity space, for the locations along the ray path indicated. The dashed curves in $(c-e)$ represent the range of the resonabce given the spred in $\mathrm{k}_{\|}$in the beam. For this discharge, the wave power is deposited near $\rho=0.5$ and $\theta_{\mathrm{p}}$ near $90 \mathrm{deg}$, with local density $1.4 \times 10^{19} \mathrm{~m}^{-3}$ and local $\mathrm{T}_{\mathrm{e}}=2.2 \mathrm{keV}$. The electron beta $\beta_{\mathrm{e}}=0.33 \%$ and the estimated optical depth is 10 .

smaller $\mathrm{v} \|$ is needed to satisfy Eq. (1). Hence, the resonance moves closer to the T-P boundary as calculated for the location shown corresponding to Fig. 4(d). For this location the FB and OK currents are approximately equal, so that $\mathrm{dI} / \mathrm{ds}$ is near zero while $(1 / \mathrm{P}) \mathrm{dP} / \mathrm{ds}$ remains large. Power deposited in this part of the ray has almost no ability to drive current. Finally, at the location shown corresponding to Fig. 4(e) the T-P boundary is osculatory to the resonance and the OK current dominates strongly, so the net $\mathrm{dI} / \mathrm{ds}$ is negative. Further along the ray $\mathrm{dI} / \mathrm{ds}$ rapidly drops to zero because the wave power is predominantly deposited in trapped electrons for which neither the FB nor the OK current drive is effective. Integrated along the ray, the net current is small because of the cancellation of the current driven along the different parts of the ray. In practice the ray bundle has a spread in $\mathrm{k}_{\|}$and in physical location, so the parts of the rays with positive and negative $\mathrm{dI} / \mathrm{ds}$ average to a small value on any particular flux surface under most conditions.

At higher $\beta_{\mathrm{e}}$ the situation is quite different. The absorption rate and optical depth at the second harmonic are proportional to $\beta_{\mathrm{e}}$, which implies that at higher $\beta_{\mathrm{e}}$ the wave will be absorbed further from the cold resonance. This reduces the trapping effects. Fig. 5 shows data from a case nearly identical to that of Fig. 4 except that the density and temperature have been doubled. The peak of absorption takes place further from the cold resonance with $\left(\omega / 2 \Omega_{e}\right)-1=0.029$ as compared with 0.018 for Fig. 4 . This 
strongly shifts the resonance away from the T-P boundary, reducing the degradation in driven FB current due to the trapping effects of the OK current.

(a)

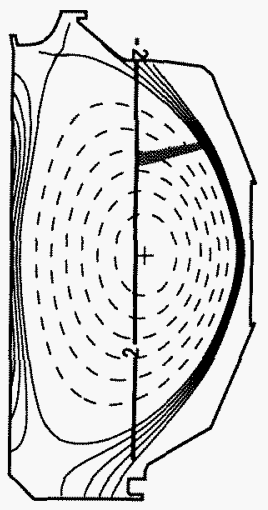

(b)

(b) 0.25

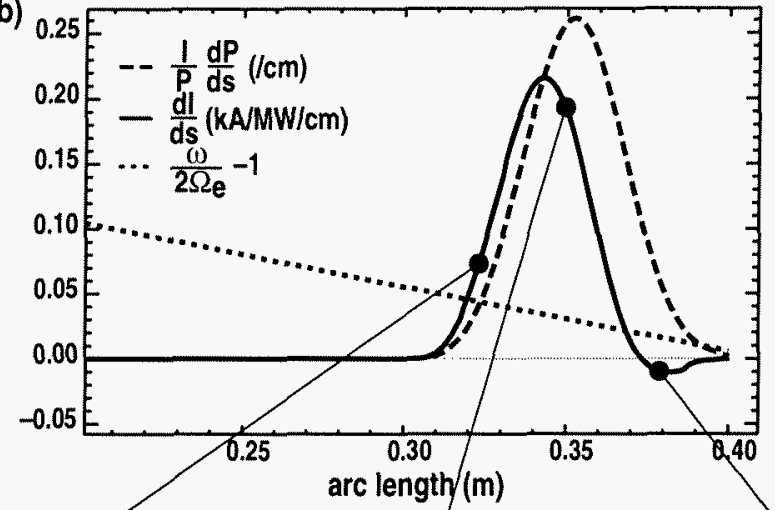

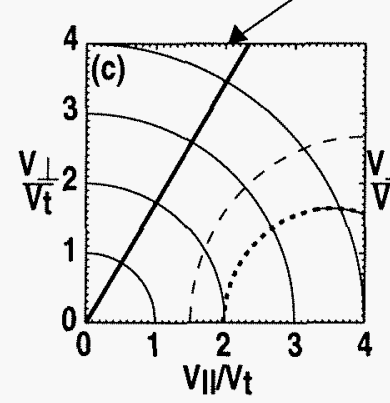
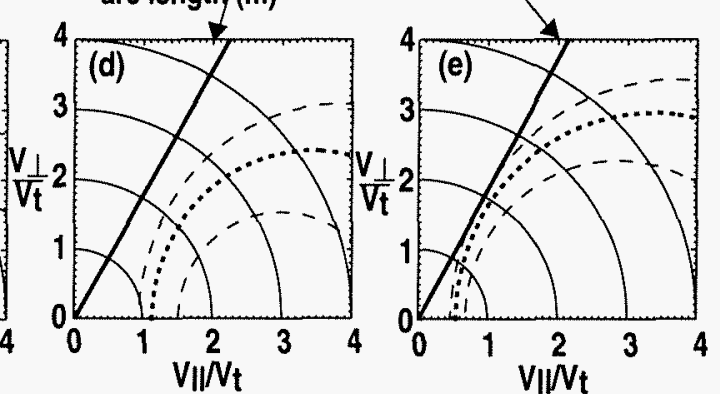

FIGURE 5. Same as Fig. 4, but the local density is $2.7 \times 10^{19} \mathrm{~m}^{-3}$ and $\mathrm{T}_{\mathrm{e}}=4.2 \mathrm{keV}$. For this case $\beta_{\mathrm{e}}=1.24 \%$ and the estimated optical depth is 38 .

For a large device like ITER the optical depth will be very large, well above 100 even for interactions near the plasma boundary where $T_{e}$ is reduced. Much of the increase in optical depth comes from the large physical size of ITER, as optical depth is proportional to $\mathrm{B} /(\mathrm{dB} / \mathrm{ds})$ which varies like the major radius. Therefore, ITER will have very little OKCD compared to FBCD. Even so, trapping effects cannot be neglected due to convective cells which set up in velocity space in response to the EC power deposition.

\section{APPLICATION OF ECCD TO AT DISCHARGES IN DIII-D}

The developing understanding of ECCD which was described above has been applied to optimize the applications of ECCD to Advanced Tokamak discharges in DIII-D. Two important applications which require off-axis ECCD are stabilization of neoclassical tearing modes and control and sustainment of current profiles with optimized magnetic shear.

Stabilization of the neoclassical tearing mode can lead to significant improvement in the performance of discharges [9]. Such a case is illustrated in Fig. 6. Here, $2 \mathrm{MW}$ of ECH power is used to drive a highly localized current near the $q=3 / 2$ surface. The aiming of the launcher optimizes the current density driven at that flux surface rather 
than the total current, since the criterion for stabilization is that the quantity $\mathrm{j}_{\mathrm{ECCD}} / \mathrm{j}_{\text {BOOTSTRAP }}$ be greater than a number of order unity rather than a condition on the total driven current. For the discharge in Fig. 6 the ECCD is applied at $3 \mathrm{~s}$ when the $3 / 2$ NTM is fully grown. The mode shrinks and disappears over a $400 \mathrm{~ms}$ period. After the mode disappears the neutral beam heating power is raised in three steps from $4 \mathrm{MW}$ to $7 \mathrm{MW}$, raising $\beta_{\mathrm{N}}$ from less than 2 when the mode is saturated to 3 . The neutron rate, a measure of plasma performance, doubles as in the last panel.

As the beta of the discharge in Fig. 6 increases, the plasma Shafranov shift increases, and the $q=3 / 2$ surface moves relative to the current drive location which is fixed by the toroidal field. So in the higher beta phase of the discharge, the ECCD is no longer well

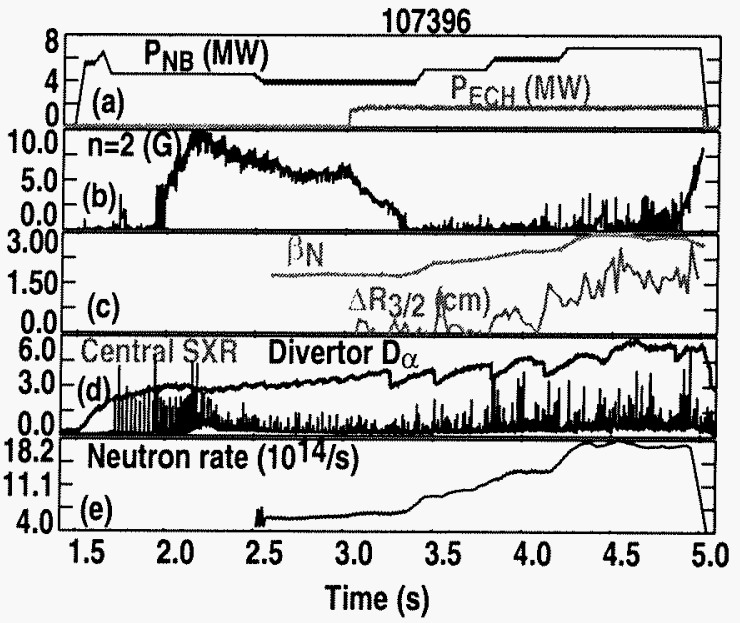

FIGURE 6. Discharge in which the $m=3 / n=2$ neoclassical tearing mode was stabilized by the application of ECCD near the $q=3 / 2$ surface at $\rho=0.54$. (a) Neutral beam and $E C H$ power, (b) amplitude of the $\mathrm{n}=2$ magnetic field fluctuation, (c) $\beta_{\mathrm{N}}$ and $\Delta \mathrm{R}_{3 / 2}$, the displacement between of the $q=3 / 2$ surface from its location when the mode is stabilized at $3.5 \mathrm{~s}$, (d) central soft $\mathrm{x}$-ray emission and $D_{\alpha}$ emission from the divertor region, and (e) the neutron emission rate, as a function of time. aligned with the rational surface and the $3 / 2$ mode eventually restarts. (Algorithms which keep the location well aligned even when the mode is not present are under development.) This observation illustrates the accuracy with which the ECCD must be aligned, and it further suggests that the actual width of the jECCD must be not much greater than the displacement which loses the stabilizing effect, or $1.5 \mathrm{~cm}$ in this case. This is consistent with the calculations of jECCD by TORAY-GA which give $2.8 \mathrm{~cm}$ in FWHM. Note that this high degree of localization is observed even in a discharge with H-mode edge with large ELMs and sawteeth [Fig. 6(d)] and with a large tearing mode present.

A second critical application of off-axis ECCD is control of the current profile as needed to obtain or sustain discharges with desired current profiles, as required by the Advanced Tokamak program. Recent results from DIII-D have demonstrated the ability to modify the current density in a high beta plasma [11]. In Fig. 7 two discharges are presented, one of which has ECCD driven by $2.5 \mathrm{MW}$ of EC power while the other discharge has heating by the same EC power but without the current drive (that is, the EC power is launched radially rather than obliquely). In both cases the EC interaction takes place near $\rho=0.4$. The evolution of the q profile is quite different in the two cases, with the co-ECCD case having much larger q0 and hence stronger central negative shear. This stronger central shear results in improved transport properties in the plasma, causing the central ion temperature to rise strongly as shown in Fig. 7(c. For this 1.2 MA discharge the fraction of the current supported noninductively is about $90 \%$, comprising $10 \% \mathrm{ECCD}, 20 \% \mathrm{NBCD}$, and $60 \%$ bootstrap current. These results validate a key motivation for the development of the ECH 
system for DIII-D: that control of the current profile will lead to improved and sustained performance.

\section{ACKNOWLEDGMENT}

Work supported by the U.S. Department of Energy under Contracts DE-AC03-99ER 54463, DE-AC0500OR22725, DE-AC02-76CH03073, and DE-FG03-99ER54463.

\section{REFERENCES}

1 Petty, C.C., Prater, R., Luce, T.C., et al., "Physics of Electron Cyclotron Current Drive on DIII-D," in Proc. of the 19th IAEA Fusion Energy Conference, Lyon, France, 2002 (International Atomic Energy Agency, Vienna).

2. Petty, C.C., Prater, R., Lohr, J., et al., Nucl. Fusion 42, 1366 (2002).

3. Luce, T.C., Lin-Liu, Y.-R., Harvey, R.W., et al., Phys. Rev. Lett. 83, 4550 (1999).

4. Matsuda, K., IEEE Trans. Plasma Sci. 17, 6 (1989).

5. Cohen, R., Phys. Fluids 30, 2442 (1987).

6. Harvey, R.W., and McCoy, M.G., in Proc. of the IAEA Technical Committee Meeting, Montreal, 1992 (International Atomic Energy Agency, Vienna, 1993) 498.

7. Prater, R., Austin, M.E., Burrell, K.H., et al., in Proc. of 18th IAEA Fusion Energy Conference, Sorrento, Italy (International Atomic Energy Agency, Vienna, 2001) paper EX8/1.

8. La Haye, R.J., Günter, S., Humphreys, D.A., et al., Phys. Plasmas 9, 2051 (2002).

9. Prater, R., La Haye, R.J., Lohr, J., et al., "Discharge Improvement Through Control of Neoclassical Tearing Modes by Localized ECCD in DIII-D," General Atomics Report GA-A24179 (2003) and submitted to Nucl. Fusion.

10. Petty, C.C., La Haye, R.J., Luce, T.C, et al., "Complete Suppression of the $m=2 / n=1$ Neoclassical Tearing Mode Using Electron Cyclotron Current Drive on DIII-D," General Atomics Report GAA24223 (2002) and submitted to Nucl. Fusion.

11. Murakami, M., Wade, M.R., DeBoo, J.C., et al., Phys. Plasmas 10, 1691 (2003).

12. Luxon, J.L., Nucl. Fusion 42, 614 (2002).

13. Lohr, J., Gorelov, Y.A., Callis, R.W., et al., in Proc. of the 12th Joint Workshop on Electron Cyclotron Emission and Electron Cyclotron Resonance Heating, Aix-en-Provence, France, 2002 (World Scientific, Singapore, 2003), 283.

14. Ellis, R., Hosea, J., Wilson, J., et al., in Radio Frequency Power in Plasmas, 14th Topical Conf., Oxnard, California 2001 (American Institute of Physics, Melville NY, 2001), 318.

15. Prater, R., Harvey, R.W., Lin-Liu, Y.-R., et al., ibid., 302.

16. Rice, B.W., Burrell, K.H., Lao, L.L., Lin-Liu, Y.R., Phys. Rev. Lett. 79, 2694 (1997). 